\title{
Substance use problem in Sudan: elephant in the room
}

\author{
Mohamed El Mahi
}

Hayat Center for Psychosocial Treatment and Rehabilitation, P.O. Box 181, Khartoum 12215 Sudan. Email: mamelmahi@ gmail.com

\section{Conflicts of interest: None.}

doi:10.1192/bji.2017.33

(c) The Author 2018. This is an Open Access article, distributed under the terms of the Creative Commons Attribution-

NonCommercial-NoDerivatives licence (http://creativecommons. -nd/4.0/), whic permits non-commercial re-use, distribution, and reproduction in any medium, provided the original work is unaltered and is properly cited. The written permission of Cambridge University Press must be obtained for comPress must be obtained for comPress must be obtained for comate a derivative work.
Alcohol and cannabis were the two main substances of misuse among certain groups in Sudan, especially young adults. Over the past decade, there has been a surge in the use of other substances, including prescribed drugs, among young people and university students in particular. There are two major shortcomings: first, in the absence of reliable data, the extent of the problem is unknown; second, the preventive and treatment interventions so far being adopted are meagre. To describe the possible causes behind the escalation of this problem and to discuss applicable measures that need to be implemented to control this rapidly growing problem. The paper will highlight the current situation of substance use in Sudan, as well as suggesting preventive measures that need to be taken to curb this problem. Research and evidence-based preventive approaches are desperately needed to aid policy makers in controlling this problem. Culture-specific treatment interventions are also necessary. The implementation of a national substance misuse control strategy is of paramount importance.

\section{Background}

Recent decades have witnessed a colossal increase in psychoactive substances use worldwide. Likewise, and owing to many socioeconomic changes in most African countries, there has been a rapid increase in the prevalence of substance use, especially among young people and university students (Tesfaye et al., 2014). Sudan is not an exception.

\section{Historical background}

The history of substance misuse in Sudan, especially of locally produced alcohol and drugs, can be traced back for many centuries. For several decades, locally made alcohol beverages and locally cultivated cannabis have been the two main substances of use among certain groups in Sudan (El Tayeb, 2004: p. 8).

Alcohol, although it has been prohibited by Sharia laws since 1983, is still illicitly used in many parts of the country. Locally produced alcoholic beverages are known as mariesa and araki. Mariesa is a beer-like beverage of low alcohol concentration, usually made from fermented grain or sorghum. It is socially accepted in some local subcultures, especially during certain societal events and festivals. On the other hand, araki, which is of much higher alcohol concentration, is obtained by distillation of palm dates and fruits. It is secretly used behind doors, e.g. at wedding ceremonies. Therefore, alcohol use has some cultural ramifications. On the other hand, cannabis, locally known as bangu and hashish, is illicitly cultivated in many areas of the country as a cash crop, adding a socioeconomic dimension to the problem.

\section{The present problem}

Over the past decade, the drug scene in Sudan has shown a rapid surge of use of many substances, including misuse of prescription drugs. Commonly misused prescription medicines among young people include tramadol (also known as strawberry or pink); benzodiazepines, e.g. clonazepam (Roche); cough syrups and antihistamines (Omer, 2016). Other substances include trihexyphenidyl (also known as kharsha or 5), anticonvulsants and neuropathic pain agents (pregabalin and gabapentin), and antipsychotic medications (e.g. quetiapine). Owing to the absence of stringent prescription and dispensing monitoring systems, most of these medicines can be obtained without prescriptions.

Solvent misuse is another evolving problem, particularly among poor children, adolescents and young adults, with fatal outcomes at times (AbdulRahim \& Al Shiekh, 2012).

However, amphetamines (lejjah), methamphetamine, opioids, cocaine and new psychoactive substances (NPS) are uncommon, probably owing to high cost. Shashabendi, for example, a more potent cannabis type that is smuggled across the Eastern borders, has dominated the drug scene recently.

\section{Discussion}

It is difficult to estimate the extent of the problem owing to the lack of reliable community-based data. One unpublished cross-sectional study showed that $84(21.3 \%)$ out of 394 medical students reported using one or more types of substances or alcohol during the academic year 2013-2014 (Ibnauf, 2016). Another study of 500 university students revealed an overall prevalence of substance misuse of one or more substances as $31 \%$; curiosity $(33.1 \%)$ was the main reason for initiation of substance use (Tarig et al, 2016). These are rather small size studies; therefore, the results cannot be extrapolated to the whole population. 
Patients with substance use problems are usually treated within general psychiatric facilities. Thus, hospital records pertaining to conditions associated with substance use are also lacking. Most of the available information is anecdotal; therefore, the exact prevalence of alcohol and substance use in Sudan remains unknown. Moreover, there is little information pertaining to the monitoring, surveillance, policy and legislation, financing or treatment system of substance use (World Health Organization, 2012).

However, some indirect indices about the problem size can be drawn from police records and reports, which show that there is a progressive annual increase in seizures resulting from substance misuse over the past decade (General Directorate of Narcotics Control, 2014). Unexpectedly, these reports show an increase in annual seizures induced by khat, which historically was not known as a substance of use in Sudan. This may be due to the great influx of refugees from eastern neighbouring countries, which are the main source of khat worldwide. Its use is most likely confined to refugees from those countries. Khat, stimulants and prescription drug misuse represent a new face of the drug problem in Sudan.

Analysis of the sociodemographic data of those arrested for drug-related crimes (2010-2014) provides greater detail about drug use in Sudan. Ninety-five per cent were male, 18-30 years old and unskilled workers of low educational level. Khartoum state, as expected, scored the highest percentage of $64 \%$ (Society Studies Center, 2015, a personal communication with the permission of the author). Moreover, a continuous increase in drug-related criminal activity is expected up to 2021, based on observed and forecasted data (General Directorate of Narcotics Control, 2014), a sign of the gravity of the situation.

This increase in substance misuse could be attributed to many intermingling factors:

(a) rapid unfavourable economic and social changes

(b) poverty: high financial gains encouraging some low-income young people to deal and use

(c) weakening of family and extended family ties and values

(d) community leaders who have not modernised their way of communication and are not keeping pace with young people's aspirations and way of thinking

(e) conflicts, displacement and semi-opened borders

(f) a marked influx of refugees, as well as foreign and expatriate Sudanese students coming to study from Gulf and Western countries, where substance use is more prevalent than in Sudan.

Sudan is surrounded by seven neighbouring countries across a vast and poorly controlled configuration of borders. Thus, it is very difficult to prevent drug smuggling and movement of drug dealers across national borders. As mentioned earlier, Sudan continues to endure many longstanding socioeconomic crises, mass displacements and civil conflicts. Substance use is an added burden that seems to pass unnoticed.

As far as supply reduction is concerned, various departments of the police and security authorities exert great effort, as reflected by a tangible increase in seizures at borders and customs ports. Cannabis is being cultivated locally for commercial purposes; thus, crop destruction and substitution followed by integrated rural development are necessary steps in order to target the root causes of illicit drug manufacture and trade (United Nations International Drug Control Programme, 1993). This important measure has only recently been enacted by drug lawenforcement authorities.

Demand reduction is an inseparable component which incorporates prevention, early detection of cases, treatment and rehabilitation. At the moment, there is only one governmental treatment centre for patients with alcohol and drug-related problems in Khartoum. There are none to be found in the country outside the capital. As mentioned previously, the problem of substance use in Sudan is twofold. First, its size is unknown. Second, preventive and treatment interventions so far being adopted are meagre.

Prevention is better than treatment by de facto and is more cost-effective. For every dollar spent on prevention, at least ten can be saved in future health, social and crime costs (National Institutes of Health, 2008). Therefore, a prevention programme based on scientific evidence is needed to target relevant ages and levels of risk in the community (United Nations Office on Drugs and Crime, 2013).

The onus is on health authorities and decision makers in other sectors to take major steps in order to curb this problem. A national drug prevalence survey is a mandatory preliminary step to discern the size of the problem. Supportive policy and a legal framework are also important complementary arrangements.

Needless to say, research and staff training are essential prerequisites to any effective prevention and treatment programme. Raising public awareness through campaigns with active community participation is an essential element for consideration. Culture-specific treatment modalities are desperately needed nationwide. These measures will not work unless they are implemented within a national drug control strategy, which, unfortunately, is a challenging goal to achieve.

\section{Conclusion}

Substance misuse in Sudan is a rapidly escalating problem of unknown size. Current efforts to reduce supply should be supplemented with measures to reduce the demand for illicit substances. A national survey to determine the extent of the problem is a mandatory first step, followed by 
the implementation of evidence-based preventive interventions. More legislative measures are needed to control and reschedule some prescription and over-the-counter medications. Treatment and rehabilitation services should be readily available and easily accessible to affected individuals. Working towards a national drug strategy and raising funds for training and research are of paramount importance.

\section{References}

AbdulRahim F. A. \& Al Shiekh A. (2012) Substance abuse and homelessness: mass methanol poisoning in Khartoum. Sudan Medical Journal, 48(1), 1-6.

El Tayeb M. T. (2004) Elindayah. Dar Azza Publishing and Distribution.

General Directorate of Narcotics Control (2014) Annual Drug Related Crime Report. MOI.

Ibnauf A. (2016) Alcohol and substance use among Sudanese medical students: a cross-sectional study. A paper presented at the 6th International Psychiatric Conference, 7-10 Oct. 2016, Khartoum, Sudan.
National Institutes of Health (2008) Principles of Drug Addiction Treatment: A Research-Based Guide, 3rd edn, p. 12. NIH.

Omer A. (2016) Profile and characteristics of the first 100 patients attended Hayat centre for psychosocial treatment and rehabilitation. A paper presented at the 6th International Psychiatric Conference, 7-10 Oct. 2016, Khartoum, Sudan.

Tarig O., Victor C., Abdulmoneim A., et al (2016) Epidemiology of substance use among university students in Sudan. Journal of Addiction, 2016(8), 2476164.

Tesfaye G., Derese A. \& Hambisa M. T. (2014) Substance use and associated factors among university students in Ethiopia: a cross-sectional study. Journal of Addiction, 2014(8), 969837.

United Nations International Drug Control Programme (1993) Alternative Development as an Instrument of Drug Abuse Control, Technical Information Paper, No. 5. UN.

United Nations Office on Drugs and Crime (2013) International Standards on Drug Use Prevention. UN.

World Health Organization (2012) Atlas: Substance Use in the Eastern Mediterranean Region. WHO.

\section{GLOBAL ECHOES} PAPER

\title{
A fish out of water: experience of working with the Māori people in New Zealand
}

\author{
Rebecca Chubb
}

Specialty Doctor in Old Age Psychiatry, North Staffordshire Combined Healthcare Trust; email rebecca.chubb1@combined. co.uk

\section{Conflicts of interest. None}

doi:10.1192/bji.2017.38

(c) The Author 2018. This is an Open Access article, distributed under the terms of the Creative Commons Attribution-

NonCommercial-NoDerivatives

NonCommercial-NoDerivatives
licence (http://creativecommons org/licenses/by-nc-nd/4.0/), which permits non-commercial re-use, distribution, and reproduction in any medium, provided the original work is unaltered and is ginal work is unaltered and is properly cited. The written permission of Cambridge University Press must be obtained for commercial re-use or in order to create a derivative work.
Experiencing life and work in an alien culture initially just felt unsettling, but it also really challenged how I perceived myself and my abilities as a doctor. Being the outsider in a group has the ability to hold an uncomfortable and scrutinising mirror up to yourself. It also offers a unique opportunity to reconsider the skills, attributes and knowledge needed to be a psychiatrist. As psychiatrists, we intellectually know that culture is integral to a personâs well-being and the care we deliver will never be truly holistic until we encompass that. However, it was only when I was the fish out of water that I truly began to appreciate its significance.

I had never felt embarrassed of being White before, but when I was in a meeting room on a forensic psychiatric unit on North Island, New Zealand, I was suddenly a very embarrassed White, British fish out of water.

The team, of which I was a member, was there to meet with the family of a Māori (the indigenous people of New Zealand) patient. As the family members walked in, they approached each of the team in turn and kissed us on the cheek. My shoulders rose towards my ears as they approached me and I could feel my stiff upper lip growing. I was every stereotype of a British person you can imagine rolled into one. Except for shaking hands (and I generally avoid that), bodily contact was not something I deemed appropriate in my line of work. I was a psychiatry trainee after all. Everybody else, including my British consultant, followed suit so... when in Rome.

The meeting opened with the social worker saying a karakia (prayer) and then a waiata (song in the Māori language). I was wholly unprepared for this; I tried to look respectful but was clearly completely ignorant. The meeting broached some very tricky topics but overall it went smoothly and ended with a further karakia. I was so ashamed that I could not participate in the customs or understand what was needed from me, something which usually I would have taken for granted. I could not pronounce their names or even begin to understand the significance of the prayers. I felt a desire to apologise for my ancestors, their colonisation and shout that I was not like that. I wanted them to see past my white face and West Midlands tone. On returning to my desk I enrolled in a Māori language course and cultural awareness day. I had of course worked with many different ethnic 